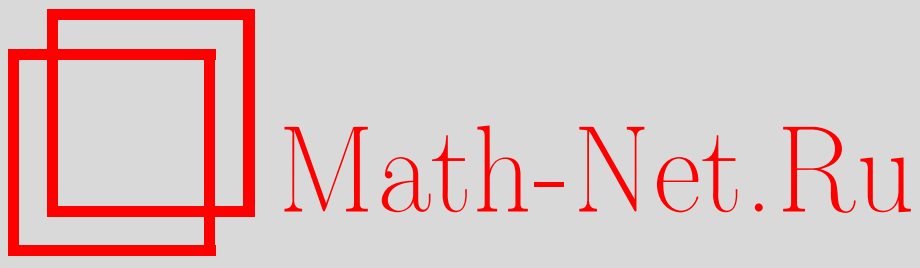

А. П. Коростелев, А. Л. Рухин, Риск по Бахадуру рекуррентных оценок многомерного параметра, Теория вероятн. и ее примен., 2000, том 45, выпуск 4, 639-656

DOI: https://doi.org/10.4213/tvp496

Использование Общероссийского математического портала Math-Net.Ru подразумевает, что вы прочитали и согласны с пользовательским соглашением http://www . mathnet.ru/rus/agreement

Параметры загрузки:

IP : 3.80 .181 .102

26 апреля 2023 г., 15:41:45

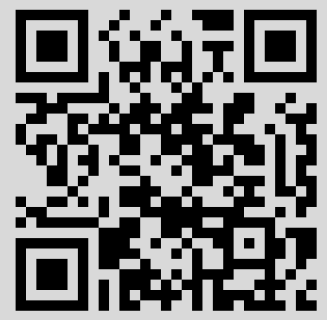




\title{
РИСК ПО БАХАДУРУ РЕКУРРЕНТНЫХ ОЦЕНОК МНОГОМЕРНОГО ПАРАМЕТРА
}

\begin{abstract}
Исследуется рекуррентная оценка многомерного параметра в ситуации, когда качество оценивания характеризуется бинарной 0-1 функцией потерь. Вычисляются вероятности больших уклонений и асимптотическая эффективность этих процедур. Результаты распространены на случай рекуррентного поискового алгоритма в полупространстве с нормальным отражением на границе.
\end{abstract}

Ключевые слова и фразы: риск по Бахадуру, большие уклонения, марковские процессы, рекуррентное оценивание, стохастическая аппроксимация.

1. Введение. Пусть независимые одинаково распределенные наблюдения $X_{1}, \ldots, X_{n}$ имеют общую плотность вероятности $f(x, \theta)$, которая зависит от неизвестного параметра $\theta$. В задаче рекуррентного оценивания параметра $\theta$ оценка $\delta_{k}$ в каждый момент $k$ может зависеть лишь от значения оценки в предыдущий момент $\delta_{k-1}$ и текущего наблюдения $X_{k}$.

Невельсон и Хасьминский [10] исследовали связь между задачами рекуррентного оценивания и стохастической аппроксимации. В этой монографии обсуждается вопрос о том, как построить последовательность рекуррентных оценок, которые были бы асимптотически эффективны при квадратичной функции потерь. Такая последовательность оценок, впервые построенная Сакрисоном [13], может рассматриваться как ряд последовательных приближений решения уравнения правдоподобия. Аналогичные процедуры были разработаны Саксом [12] и Фабианом [3] при разных условиях регулярности. Вероятности больших уклонений были использованы Коростелевым [6] для анализа условий сходимости процедур стохастической аппроксимации и рекуррентного оценивания. Рухин [11] изучал пошаговую или рекуррентную задачу принятия решений.

В настоящей работе мы исследуем асимптотическое поведение риска по Бахадуру при рекуррентном оценивании. Пусть $D_{\theta}$ является открытым множеством, содержащим $\theta$. Поведение оценки $\delta_{n}=\delta_{n}\left(X_{1}, \ldots, X_{n}\right)$

\footnotetext{
*Department of Mathematics, Wayne State University, Detroit MI 48202, USA.

** Department of Mathematics and Statistics, UMBC, Baltimore MD 21250, USA; e-mail: rukhin@pc19.math.umbc.edu
} 
параметра $\theta$ будет измеряться вероятностью (так называемый риск по Бахадуру)

$$
\beta_{n}\left(\delta_{n}, \theta, D_{\theta}\right)=n^{-1} \ln \mathbf{P}_{\theta}\left\{\delta_{n} \notin D_{\theta}\right\}
$$

Если $D_{\theta}$ - шар радиуса $c$ с центром в $\theta$, то мы получаем классическую вероятностную характеристику $\delta_{n}$, которой часто описывают качество доверительных интервалов. Она также является основой теории асимптотической эффективности по Бахадуру [1].

Предположим, что $\psi(x, \vartheta)$ - измеримая вектор-функция, зависящая от $x, \vartheta \in \mathbf{R}^{p}$. (Обычно $\psi$ - это градиент выпуклой функции.) Определим оценку $\delta_{k}$ следующей рекуррентной формулой:

$$
\delta_{k}=\delta_{k-1}+\frac{J^{-1} \psi\left(X_{k}, \delta_{k-1}\right)}{k}, \quad k=1,2, \ldots,
$$

с начальным условием $\delta_{0}$. Здесь мы полагаем, что $J$ - невырожденная матрица нормализации, которая выбирается на основе знания $\psi$ и $f$.

Оценку (1) можно интерпретировать как рекуррентную оценку минимального контраста или М-оценку. Эта оценка обладает многими замечательными свойствами классической М-оценки, определяемой как корень уравнения

$$
\sum_{k=1}^{n} \psi\left(X_{k}, \vartheta\right)=0 .
$$

В том случае, когда $\psi(x, \vartheta)=\frac{\partial}{\partial \vartheta} \ln f(x, \vartheta)$, а $J$ является информационной матрицей Фишера, соотношение (2) приводит к упомянутой выше оценке Сакрисона - рекуррентному аналогу оценки максимального правдоподобия.

Когда параметрическое семейство $f(x, \vartheta)$ известно, такой выбор $\psi$ оптимален. Однако иной выбор функции $\psi$ может быть интересен при не полностью определенной модели или при робастном оденивании, например, когда желательно использовать ограниченные функции.

В одномерном случае, когда $p=1, D_{\theta}=(\theta-c, \theta+c), c>0$, и $\psi, J$ удовлетворяют некоторым условиям регулярности, явная величина предельного значения

$$
\lim _{n \rightarrow \infty} \beta_{n}\left(\delta_{n}, \theta, D_{\theta}\right)=-V_{\theta}(x)
$$

была найдена в [9].

В п. 2 в предположениях регулярности найдено предельное выражение для $\beta_{n}\left(\delta_{n}, \theta, D_{\theta}\right)$ при произвольной размерности $p$. Как обычно в теории больших уклонений (см. [5]), формула для $\beta_{n}\left(\delta_{n}, \theta, D_{\theta}\right)$ получена в форме минимального значения интегрального функционала. Особенностью задачи в нашем случае является то, что этот функционал неоднороден по времени. 
Как правило, в рекуррентном оценивании траектория $\delta_{k}$ сходится к $\theta$ при $k \rightarrow \infty$, где точка $\theta$ может быть интерпретирована как точка экстремума некоторой задачи оптимизации. Если эта точка экстремума находится в области, отличной от всего пространства $\mathbf{R}^{p}$, то имеет смысл ограничить траектории поисковой процедуры (2) той же самой областью.

В п. 3 мы изучаем ситуацию, когда поиск ограничен полупространством и рекуррентная оценка (2) модифицируется условием нормального отражения на границе области. Пример линейной системы при гауссовском шуме рассмотрен в п. 2. Он может быть интересен для сравнения с классической асимптотической нормальностью. В п. 3 исследуется более сложный пример, иллюстрирующий несколько неожиданное поведение модифицированной оценки с отражением на границе. Доказательства всех результатов отнесены в приложение (п. 4).

2. Рекуррентные процедуры и риск по Бахадуру. Для изучения асимптотического поведения риска по Бахадуру (1) при заданной области $D_{\theta}$ нам потребуются некоторые условия. Во-первых, мы предполагаем априори, что $\theta$ принадлежит кубу $[-A, A]^{p}, A>0$, и существует некий больший куб $[-K, K]^{p}$ такой, что при всех $\theta \in[-A, A]^{p}$ имеет место включение: $D_{\theta} \subseteq[-K, K]^{p}$. Введем обозначения

$$
\begin{gathered}
m_{\theta}(\vartheta)=J^{-1} \mathbf{E}_{\theta} \psi(X, \vartheta)=J^{-1} \int_{\mathbf{R}^{p}} \psi(x, \vartheta) f(x, \theta) d x, \quad \vartheta \in \mathbf{R}^{p} \\
v_{k}(x, z)=z+\frac{J^{-1} \psi(x, z)}{k}, \quad k=1,2, \ldots ; \xi, z \in \mathbf{R}^{p}
\end{gathered}
$$

где $J$ - невырожденная матрица.

П р е д п о о ж е н и 1 . Функция $m_{\theta}(\cdot)$ задает гладкое векторное поле в $\mathbf{R}^{p}$. Для любого фиксированного $\theta \in[-A, A]^{p}$ точка $\vartheta=\theta$ является единственным устойчивым положением равновесия динамической системы $\dot{x}_{t}=m_{\theta}\left(x_{t}\right)$ в кубе $[-K, K]^{p}$; для любого начального условия $x_{0} \in[-K, K]^{p}$ траектория $x_{t}, t \geqslant 0$, остается в этом кубе и $x_{t} \rightarrow \theta$ при $t \rightarrow \infty$.

Зададим модифицированную рекуррентную процедуру (2) посредством усечения:

$$
\delta_{k}= \begin{cases}v_{k}\left(X_{k}, \delta_{k-1}\right), & \text { если } v_{k}\left(X_{k}, \delta_{k-1}\right) \in[-K, K]^{p} \\ 0 & \text { в противном случае }\end{cases}
$$

при нулевом начальном условии $\delta_{0}=0$. Усечение в (4) принимает во внимание возможную неединственность решения уравнения $m_{\theta}(\vartheta)=0$ во всем пространстве $\mathbf{R}^{p}$. В предположении 1 такое решение единственно в кубе $[-K, K]^{p}$. 
П ре д п оложе н и е 2. Кумулянта

$$
G_{\theta}(v, \vartheta)=\ln \int_{\mathbf{R}^{p}} \exp \left\{v^{T} J^{-1} \psi(x, \vartheta)\right\} f(x, \theta) d x
$$

конечна для любого $v \in \mathbf{R}^{p}$ и липшицева по $\vartheta, \vartheta \in[-K, K]^{p}$, равномерно относительно $\theta \in[-A, A]^{p}$.

Определим

$$
H_{\theta}(u, \vartheta)=\sup _{v}\left[u^{T} v-G_{\theta}(v, \vartheta)\right], \quad u \in \mathbf{R}^{p},
$$

как преобразование Лежандра функции $G_{\theta}(v, \vartheta)$ по переменной $v$. Следующее предположение позволяет применить теорию больших уклонений для марковских процессов, следуя работе [14].

П р е д п оло же н и е 3 . Функция $H_{\theta}(u, \vartheta)$ конечна при всех $u \in \mathbf{R}^{p}$ и дважды непрерывно дифференцируема по $u$ на любом компактном множестве, причем равномерно по $\vartheta \in[-K, K]^{p}$ ил $\theta \in[-A, A]^{p}$.

Введем множество вектор-функций $\varphi(t)=\left(\varphi_{1}(t), \ldots, \varphi_{p}(t)\right)^{T}$ :

$$
\begin{aligned}
\Phi(x)=\{ & \varphi(t), t \in(-\infty, 0]: \varphi(t) \text { кусочно абсолютно непрерывны; } \\
& \varphi \in[-K, K]^{p} ; \text { в каждой точке разрыва } \varphi(t-0) \in \partial[-K, K]^{p}, \\
& \left.\varphi(t+0)=0 ; \quad \varphi(0)=x \in[-K, K]^{p}\right\} .
\end{aligned}
$$

Функции из $\Phi(x)$ призваны отразить свойства траекторий системы (4) в иной шкале времени: они начинаются в $t=-\infty$ и заканчиваются в момент времени $t=0$ в заданной точке $x$.

Для неотришательных $\tau$ определим функиионал действия $I_{(\tau, 0)}(\varphi)$ как

$$
I_{(\tau, 0)}(\varphi)=\int_{\tau}^{0} e^{t} H_{\theta}(\dot{\varphi}(t), \varphi(t)) d t
$$

где $\dot{\varphi}(t)=\left(\frac{d}{d t} \varphi_{1}(t), \ldots, \frac{d}{d t} \varphi_{p}(t)\right)^{T}$. Следующая функция, называемая квазипотенциалом, описывает асимптотическое поведение риска по Бахадуру рекуррентной процедуры (1):

$$
V_{\theta}(x)=\inf _{\varphi \in \Phi(x)} I_{(-\infty, 0)}(\varphi), \quad x \in[-K, K]^{p}, \theta \in[-A, A]^{p} .
$$

Отметим, что $V_{\theta}(x) \geqslant 0$ и $V_{\theta}(\theta)=0$. Действительно, функция $H_{\theta}(u, \vartheta)$ выпукла по $u$, неотрицательна и $H_{\theta}(0, \theta)=0$ (см. [5, раздел 5.1]).

Теорема 1. B предположениях 1-3 риск по Бахадуру (1) рекуррентной оченки (4) сходится равномерно относительно $\theta \in[-A, A]^{p}$ :

$$
\lim _{n \rightarrow \infty} \beta_{n}\left(\delta_{n}, \theta, D_{\theta}\right)=-\inf _{x \notin D_{\theta}} V_{\theta}(x) .
$$


3 а м е ч а н и е 1. Множества уровней $W_{\theta}(\lambda)$ квазипотенциала, определяемые для любого $\lambda \geqslant 0$ как

$$
W_{\theta}(\lambda)=\left\{x: V_{\theta}(x) \leqslant \lambda\right\},
$$

играют важную роль в описании асимптотического поведения процедуры (4). Действительно, формула (5) может быть переписана в виде

$$
\lim _{n \rightarrow \infty} \beta_{n}\left(\delta_{n}, \theta, D_{\theta}\right)=\inf \left\{\lambda: D_{\theta}^{c} \cap W_{\theta}(\lambda) \neq \varnothing\right\},
$$

где $D_{\theta}^{c}$ обозначает дополнение к $D_{\theta}$. В частности, если множество $W_{\theta}^{c}(\lambda) \cap[-K, K]^{p}$ непусто, то

$$
\lim _{n \rightarrow \infty} \beta_{n}\left(\delta_{n}, \theta, W_{\theta}(\lambda)\right)=-\lambda,
$$

т.е. $W_{\theta}(\lambda)$ очерчивает множество равных вероятностей больших уклонений.

Асимптотическое поведение $\beta_{n}\left(\delta_{n}^{*}, \theta, D_{\theta}\right)$ оценки максимального правдоподобия $\delta_{n}^{*}$ было детально изучено Бахадуром [1]. В наших обозначениях результат его теоремы 6.1 может быть сформулирован так:

$$
\lim _{n \rightarrow \infty} \beta_{n}\left(\delta_{n}, \theta, W_{\theta}(\lambda)\right)=-\lambda\left[1+o_{\lambda}(1)\right]
$$

где $o_{\lambda}(1) \rightarrow 0$ при $\lambda \rightarrow 0$, а

$$
W_{\theta}(\lambda)=\left\{x \in \mathbf{R}^{p}: \frac{1}{2}(x-\theta)^{T} I_{f}(\theta)(x-\theta) \leqslant \lambda\right\}
$$

- эллипсоид, определяемый информационной матрицей Фишера $I_{f}(\theta)$.

Как было показано в [4], [5], малые значения $\lambda$ соответствуют гауссовской аппроксимации вероятностей больших уклонений в (4). Следующий пример иллюстрирует некоторые детали.

П р и м е р 1. Если $\vartheta-\theta$ мало, то $m_{\theta}(\vartheta) \approx-\mathscr{K}[\theta-\theta]$, где $-\mathscr{K}=$ $\left.\nabla_{v} m_{\theta}(v)\right|_{v=\theta}$. Пусть

$$
F=F(\theta)=\int_{-\infty}^{\infty} J^{-1} \psi(x, \theta) \psi^{T}(x, \theta)\left[J^{T}\right]^{-1} f(x, \theta) d x
$$

$F$ предполагается положительно определенной (невырожденной, симметричной) матрицей. Разложение Тейлора функции $G_{\theta}(v, \vartheta)$ в точке $v=0$, $\vartheta=\theta$ показывает, что

$$
G_{\theta}(v, \vartheta) \approx-v^{T} \mathscr{K}[\vartheta-\theta]+\frac{1}{2} v^{T} F v
$$

Это и является аппроксимацией возмушенной динамической системы $\dot{x}(t)=-\mathscr{K}[x(t)-\theta], t \geqslant 0$, гауссовским белым шумом с ковариационной матрицей $F$.

Мы примем (6) за основу при явном вычислении квазипотенциала. Следующее предложение показывает, что теорема 1 согласуется с классическими результатами по асимптотической нормальности (см. [10]). 
Предложение 1. Пусть $A=K=\infty$, и пусть функция $G_{\theta}(v, \vartheta)$ задается правой частью равенства (6). Предположим, ито матрича $-\mathscr{K}+\frac{1}{2} \mathbf{I}$, где I обозначает единичную матричу, устойчива. Тогда

$$
V_{\theta}(x)=\frac{1}{2}(x-\theta)^{T} \mathscr{S}^{-1}(x-\theta),
$$

zde

$$
\mathscr{S}=\int_{-\infty}^{0} e^{t} \exp \{t(\mathscr{K}-\mathbf{I})\} F \exp \{t(\mathscr{K}-\mathbf{I})\} d t .
$$

Если $\mathscr{K}$ и $F$ коммутируют, то $\mathscr{S}=(2 \mathscr{K}-\mathbf{I})^{-1} F$.

3 а м е ч а н и е 2 . Если функция $G_{\theta}(v, \vartheta)$ со своими двумя производными по $v$ может быть аппроксимирована правой частью (6) при $\vartheta \rightarrow \theta$ и $v \rightarrow 0$, то аналог предложения 1 справедлив для малых значений $\lambda$. Все рассмотрения при этом локальны, усечение в (4) существенно, и, вообще говоря, нельзя полагать $A=K=\infty$.

3 а м е ч а н и е 3 . Если матрица $-\mathscr{K}+\frac{1}{2} \mathbf{I}$ имеет собственные значения с положительной вещественной частью, то для любого шара $B_{c}=\left\{x \in \mathbf{R}^{p}:(x-\theta)^{T}(x-\theta) \leqslant c\right\}$ асимптотическое поведение $\beta_{n}\left(\delta_{n}, \theta, B_{c}\right)$ вырождено:

$$
\lim _{n \rightarrow \infty} \beta_{n}\left(\delta_{n}, \theta, B_{c}\right)=0
$$

(ср. с одномерным случаем в работе [9]).

3 а м е ч а н и е 4 . Экстремаль $\varphi(t)$, дающая минимум функционалу действия $I_{(-\infty, 0)}(\varphi)$ в предложении 1 , ограничена при условии, что матрица $-\mathscr{K}+\mathbf{I}$ устойчива. Более того, в этом случае $\varphi(t)$ и все ее производные исчезающе малы, когда $x \rightarrow \theta$.

3. Рекуррентная процедура с отражением. Траектория $\delta_{k}$ рекуррентной процедуры (4) типично следует за траекторией динамической системы $\dot{x}_{t}=m_{\theta}\left(x_{t}\right)$ при $k \rightarrow \infty$. В частности, если $m_{\theta}(\vartheta)=\nabla Q_{\theta}(\vartheta)$ является градиентом выпуклой функции $Q_{\theta}$, как в случае максимума правдоподобия при логарифмически выпуклой плотности вероятностей, то траектории (4) нацелены на поиск точки максимума функции $Q_{\theta}$.

Связь между этим поисковым методом и алгоритмом КифераВольфовица хорошо известна в стохастической аппроксимации (см. [10]).

Усеченная процедура (4) ограничена кубом $[-K, K]^{p}$. Во мноrиx приложениях область поиска дополнительно ограничена еще и некоторой областью. Для простоты изложения мы предполагаем, что эта дополиительная область является полупространством $\mathbf{R}_{+}^{p}=\{x=$ $\left.\left(x_{1}, \ldots, x_{p}\right)^{T}, x_{1} \geqslant 0\right\}$, п мы ищем условный максимум $Q_{\theta}(\vartheta)$ при условии, что $\vartheta \in[-K, K]^{p} \cap \mathbf{R}_{+}^{p}$. В дальнейшем мы предполагаем, что семейство функций $Q_{\theta}$ параметризовано таким образом, что $\theta$ совпадает с точкой условного максимума. 
Модифицируем рекуррентную оценку $\delta_{k}$ в (4) так, чтобы ее траектории оставались в $\mathbf{R}_{+}^{p}$. Для того чтобы вынудить траектории $\delta_{k}$, $k=1,2, \ldots$, оставаться в этом полупространстве, мы определяем следуюшую рехуррентную прочедуру с отражением:

$$
\tilde{\delta}_{k}= \begin{cases}v_{k}\left(X_{k}, \tilde{\delta}_{k-1}\right), & \text { если } e_{1}^{T} v_{k}\left(X_{k}, \tilde{\delta}_{k-1}\right) \geqslant 0 \\ v_{k}\left(X_{k}, \widetilde{\delta}_{k-1}\right)-2\left(e_{1}^{T} v_{k}\left(X_{k}, \tilde{\delta}_{k-1}\right)\right) e_{1} & \text { в противном случае }\end{cases}
$$

где $v_{k}(x, z)=z+(k J)^{-1} \psi(x, z)$ обозначает то же, что и в $(4), \widetilde{\delta}_{k} \in$ $[-K, K]^{p} \cap \mathbf{R}_{+}^{p}$ и $e_{1}=(1,0, \ldots, 0)^{T}$. Определение $(7)$ должно быть дополнено начальным условием $\widetilde{\delta}_{0} \in[-K, K]^{p} \cap \mathbf{R}_{+}^{p}$.

Основные определения и предположения п. 2 должны быть модифицированы. Положим

$$
\tilde{m}_{\theta}(\vartheta)= \begin{cases}m_{\theta}(\vartheta)-\left(e_{1}^{T} m_{\theta}(\vartheta)\right) e_{1}, & \text { если } e_{1}^{T} \vartheta=0, \quad e_{1}^{T} m_{\theta}(\vartheta)<0 \\ m_{\theta}(\vartheta) & \text { в противном случае. }\end{cases}
$$

Заметим, что $\tilde{m}_{\theta}(\vartheta)$ - проекция $m_{\theta}(\vartheta)$ на границу $\partial \mathbf{R}_{+}^{p}$, если $\vartheta \in \partial \mathbf{R}_{+}^{p}$ и $m_{\theta}(\vartheta)$ направлено вовне. Векторное поле $\widetilde{m}_{\theta}(\vartheta)$ может быть разрывным на границе $\partial \mathbf{R}_{+}^{p}$.

П р ед положени е $1^{\prime}$. Пусть точка $\vartheta=\theta \in \mathbf{R}_{+}^{p}$ является единственным устойчивым положением равновесия динамической системы $\dot{x}_{t}=\widetilde{m}_{\theta}\left(x_{t}\right)$ в $[-K, K]^{p} \cap \mathbf{R}_{+}^{p}$, для любого начального условия $x_{0} \in[-K, K]^{p} \cap \mathbf{R}_{+}^{p}$ траектория $x_{t}$ остается в этой области и $x_{t} \rightarrow \theta$ при $t \rightarrow \infty$.

Пусть $H_{\theta}(u, \vartheta)$ определено так же, как и в предыдущем пункте. Положим

$$
\begin{gathered}
\widetilde{H}_{\theta}(u, \vartheta)= \begin{cases}\min _{\alpha>0} H_{\theta}\left(u-\alpha e_{1}, \vartheta\right), & \text { если } e_{1}^{T} \vartheta=e_{1}^{T} u=0, \\
H_{\theta}(u, \vartheta) & \text { в противном случае; }\end{cases} \\
\tilde{I}_{(\tau, 0)}(\varphi)=\int_{\tau}^{0} e^{t} \tilde{H}_{\theta}(\dot{\varphi}(t), \varphi(t)) d t, \quad \tau \leqslant 0 .
\end{gathered}
$$

Для любого $x \in \mathbf{R}_{+}^{p}$ введем множество

$$
\Phi_{+}(x)=\Phi(x) \bigcap\left\{\varphi(t) \in \mathbf{R}_{+}^{p}, t \leqslant 0\right\}
$$

абсолютно непрерывных функций и модифицированный квазипотенциал

$$
\tilde{V}_{\theta}(x)=\inf _{\varphi \in \Phi_{+}(x)} \tilde{I}_{(-\infty, 0)}(\varphi), \quad x \in \mathbf{R}_{+}^{p} .
$$

Теорема 2. При выполнении предположений 1', 2 и 3 риск по Бахадуру (1) рекуррентной прочедуры с отражением (7) имеет следуюuую acusnmomuкy:

$$
\lim _{n \rightarrow \infty} \beta_{n}\left(\tilde{\delta}_{n}, \theta, D_{\theta}\right)=-\inf _{x \notin D_{\theta}} \tilde{V}_{\theta}(x)
$$


Мы опускаем доказательство этой теоремы, поскольку оно является комбинацией доказательства теоремы 1 и принципа Лапласа для случайного блуждания с отражением, изученного в гл. 7 монографии [2].

Как и в замечании 1 , можно ввести множества уровней модифицированного квазипотенциала

$$
\widetilde{W}_{\theta}(\lambda)=\left\{x \in \mathbf{R}_{+}^{p}, \widetilde{V}_{\vartheta}(x) \leqslant \lambda\right\}, \quad \lambda \geqslant 0 .
$$

Ввиду (8) эти множества ограничивают области равных вероятностей уклонений $\widetilde{\delta}_{n}-\theta$ для больших значений $n$. Теперь мы обратимся $\mathbf{x}$ специальному случаю теоремы 2.

П р и м е р 2. Пусть $Q_{\theta}(x)$ является семейством выпуклых функций. Предположим, что $\psi\left(X_{k}, \tilde{\delta}_{k-1}\right)=\nabla Q_{\theta}\left(\tilde{\delta}_{k-1}\right)+X_{k}-$ это измерение градиента $\nabla Q_{\theta}\left(\tilde{\delta}_{k-1}\right)$ в точке $x=\tilde{\delta}_{k-1}$ со случайным шумом $X_{k}$. Пусть $X_{k}, k=1,2, \ldots$, являются независимыми случайными гауссовскими векторами с нулевым средним и матрицей ковариаций $\Sigma$. Выберем в $(p-1)$-мерном пространстве, $x_{1}=0$, базис, в котором последние $p-1$ координат нашего гауссовского вектора независимы. В этих координатах ненулевые элементы матрицы $\Sigma$ равны $\Sigma_{i i}=\sigma_{i}^{2}, i=1, \ldots, p$, и $\Sigma_{1 j}=\Sigma_{j 1}=\rho_{j} \sigma_{1} \sigma_{j}, j=2, \ldots, p$. Здесь $\sigma_{i}^{2}$ - дисперсия $i$-й координаты, а $\rho_{j}$ - это коэффициент корреляции первой и $j$-й координат.

Интересные эффекты можно ожидать, если точка условного максимума находится на границе $\partial \mathbf{R}_{+}^{p}$. Для того чтобы проиллюстрировать возможные эффекты, введем квадратичную функцию

$$
Q_{\theta}(\vartheta)=-\frac{1}{2} a_{1}\left(\vartheta_{1}+1\right)^{2}-\frac{1}{2} \sum_{i, j=2}^{p} A_{i j}\left(\vartheta_{i}-\theta_{i}\right)\left(\vartheta_{j}-\theta_{j}\right), \quad a_{1}>1 .
$$

Здесь $A=\left\{A_{i j}\right\}, i, j=2, \ldots, p,-$ симметричная $(p-1) \times(p-1)$-матрица, причем $(A-\mathbf{I})$ положительно определена. Мы полагаем, что $\theta$ имеет нулевую первую координату, т.е. $\theta=\left(0, \theta_{2}, \ldots, \theta_{p}\right)^{T}$. Динамическая система $\dot{x}_{t}=\tilde{\nabla} Q_{\theta}\left(x_{t}\right)$ имеет устойчивое положение равновесия в $\theta \in \partial \mathbf{R}_{+}^{p}$, и градиент в этой точке невырожден и направлен вовне: $\nabla Q_{\theta}(\theta)=\left(-a_{1}, 0, \ldots, 0\right)^{T}$.

Для любого $x=\left(x_{1}, x_{2}, \ldots, x_{p}\right)^{T} \in \mathbf{R}^{p}$ введем $(p-1)$-мерный вектор $\bar{x}=\left(x_{2}, \ldots, x_{p}\right)^{T}$ такой, что $x^{T}=\left(x_{1}, \bar{x}^{T}\right)$ и $\theta^{T}=\left(0, \bar{\theta}^{T}\right)$. В этих обозначениях

$$
Q_{\theta}(\vartheta)=-\frac{1}{2} a_{1}\left(\vartheta_{1}+1\right)^{2}-\frac{1}{2}(\bar{v}-\bar{\theta})^{T} A(\bar{v}-\bar{\theta}) .
$$

Через $\mathbf{R}^{2}=\rho_{2}^{2}+\cdots+\rho_{p}^{2}$ мы обозначим множественный коэффициент корреляции, предполагая, что $\mathbf{R}^{2}<1$. Пусть $(p-1)$-мерный вектор $\mathbf{s}$ определяется как

$$
\mathbf{s}=\left(\frac{\rho_{2}}{\sigma_{2}}, \ldots, \frac{\rho_{p}}{\sigma_{p}}\right)^{T}
$$


Введем диагональную матрищу $\bar{F}=\operatorname{diag}\left(\sigma_{2}^{-2}, \ldots, \sigma_{p}^{-2}\right)$ размера $(p-1) \times$ $(p-1)$ и положим

$$
\overline{\mathscr{S}}=\int_{-\infty}^{0} e^{t} \exp \{t(A-\mathbf{I})\} \bar{F} \exp \{t(A-\mathbf{I})\} d t
$$

Нашей целью является изучение асимптотического поведения области $\widetilde{W}_{\theta}(\lambda)$ при $\lambda \rightarrow 0$. Несколько неожиданно, что асимптотически эта область не зависит от коэффициентов корреляции $\rho_{j}, j=2, \ldots, p$. Следуюшее предложение 2 показывает, что эта зависимость может внести лишь исчезаюше малый член в квазипотенциал при малых значениях $\lambda$. Несколько перегружая обозначения, мы будем понимать $\bar{V}_{(0, \bar{\theta})}\left(x_{1}, \bar{x}\right)$ Kak $\bar{V}_{\theta}(x)$.

Предложение 2. При сделанных вычше предположениях выполнено

$$
\widetilde{V}_{(0, \bar{\theta})}(x)=\widetilde{V}_{(0, \bar{\theta})}\left(x_{1}, \bar{x}\right)=\lambda\left[1+o_{\lambda}(1)\right], \quad o_{\lambda}(1) \rightarrow 0 \quad n p u \lambda \rightarrow 0
$$

при условии, что

$$
\frac{2 a_{1} x_{1}}{\sigma_{1}^{2}}+\frac{1}{2}(\bar{x}-\bar{\theta})^{T \bar{S}^{-1}}(\bar{x}-\bar{\theta})=\lambda, \quad \lambda \geqslant 0, x_{1} \geqslant 0 .
$$

3 а м е ч а и е 5. Физическое объяснение результатов предложения 2 следуюшее: частица, движение которой описывается рекуррентным соотношением (7), «прижимается» к границе значительной силой и не может уйти от границы на большой период времени. Экстремали функционала в тангенциальном и нормальном направлениях «существуют» в разных шкалах времени: первая координата отличается от нуля лишь на коротком промежутке времени порядка $O(\lambda)$. В определенном смысле нормальная и тангенциальная координаты почти независимы на уровне вероятностей больших уклонений при малых значениях $\lambda$. Отметим, что в традиционной асимптотике скорость сходимости в тангенциальном направлении есть $O(1 / \sqrt{n})$, в то время как скорость сходимости в нормальном направлении есть $O(1 / n)$ при $n \rightarrow \infty$ (см. [6]).

4. Приложение. Д ок а а т ел в с т о т е о ем ы 1. Как и в одномерном случае (см. [9]), введем переменную $s$ для «нового времени» посредством

$$
s=s(k)=\sum_{i=1}^{k} \frac{1}{i}=(1+o(1)) \ln k
$$

и положим $s(0)=0$. Отметим, что $s$ принимает значения из дискретного множества $\Sigma=\left\{0,1,1+\frac{1}{2}, \ldots\right\}$. Обозначим через $\left(Y(s), \mathbf{P}_{s_{0}, y}\right)$, 
$s \in \Sigma$, марковский процесс, получаемый из $\delta_{k}$ в (4) путем замены времени: $Y(s(k))=\delta_{k}, k=1,2, \ldots, Y\left(s_{0}\right)=0$, и через $\mathbf{P}_{s_{0}, y}$ семейство вероятностных распределений, связанных с начальными условиями $Y\left(s_{0}\right)=y \in[K, K]^{p}, s_{0} \in \Sigma$.

Для пропзвольного $\tau<0$ определим последовательность марковских пропессов $Y^{(n)}(t)$ как

$$
Y^{(n)}(t)=Y(s(n)+t), \quad \tau \leqslant t \leqslant 0, s(n)+t \in \Sigma, n=1,2, \ldots .
$$

Поведение вероятностей больших уклонений для семейства $Y^{(n)}(t)$ будет описываться функционалом действия $I_{(\tau, 0)}(\varphi)$ с нормирующим множителем $n$, если мы покажем, что нормализованная производяшая функция моментов

$\widetilde{G}_{\theta}(t, v, \vartheta)=\lim _{n \rightarrow \infty} \frac{1}{n(s(k+1)-s(k))} \ln \mathbf{E}_{s(k), \vartheta}\left(\exp \left\{n v^{T}(Y(s(k+1))-\vartheta)\right\}\right)$

имеет надлежащий предел. Здесь величина $t=s(k)-s(n) \leqslant 0$ задана так, что $k / n=\left[1+o_{n}(1)\right] e^{t}$, где $o_{n}(1) \rightarrow 0$ при $n \rightarrow \infty$. Применяя эту формулу, мы получаем

$$
\begin{aligned}
\widetilde{G}_{\theta}(t, v, \vartheta) & =\lim _{n \rightarrow \infty} \frac{k}{n} \ln \int \exp \left(n k^{-1} v^{T} J^{-1} \varphi(x, \vartheta)\right) f(x, \theta) d x \\
& =e^{t} G_{\theta}\left(e^{-t} v, \vartheta\right) .
\end{aligned}
$$

Преобразование Лежандра от $\widetilde{G}_{\theta}(t, v, \vartheta)$ по $v$ есть $e^{t} H_{\theta}(u, \vartheta)$. Результаты Вентцеля (см. [14, гл. 3]), показывают, что вероятности больших уклонений $Y^{(n)}(t)$ на любом конечном интервале $T \leqslant t \leqslant 0, T<0$, определяются функционалом $I_{(T, 0)}(\varphi)$. Выберем фиксированное $T, T<0$, и положим без ограничения общности, что $s(n)+T \in \Sigma$. Определим

$$
\|Y-\varphi\|_{\infty}=\max _{T \leqslant t \leqslant 0}|Y(s(n)+t)-\varphi(t)|
$$

как максимальное отклонение $Y^{(n)}(t)$ от $\varphi(t)$, где $Y^{(n)}$ и $\varphi$ имеют общее начальное значение $Y^{(n)}(T)=\varphi(T)=y$. Как показано в [14], для сколь угодно малого $\varepsilon_{0}>0$ справедлива следующая нижняя граница:

$$
\liminf _{n \rightarrow \infty} n^{-1} \ln \mathbf{P}_{s(n)+T, y}\left\{\|Y-\varphi\|_{\infty}<\varepsilon_{0}\right\} \geqslant-I_{(T, 0)}(\varphi) .
$$

Для сколь угодно малого $\varepsilon>0$ выберем $\varphi^{(0)}=\varphi_{\theta}^{(0)}(t)$ так, чтобы $\varphi_{\theta}^{(0)}(0) \notin D_{\theta}$, т.е. евклидово расстояние $\operatorname{dist}\left(\varphi_{\theta}^{(0)}(0) ; D_{\theta}\right)=\varepsilon_{0}>0$, и $I_{(-\infty, 0)}\left(\varphi^{(0)}\right) \leqslant \inf _{x \notin D_{\theta}} V_{\theta}(x)+\varepsilon$. Для заданного значения $y$ определим

$$
\varphi^{(1)}(t)= \begin{cases}\varphi^{(0)}(t), & \text { если } t \in\left[\frac{T}{2}, 0\right], \\ y+\frac{y-\varphi^{(0)}(T / 2)}{T / 2}\left(t-\frac{T}{2}\right), & \text { если } t \in\left[T, \frac{T}{2}\right] .\end{cases}
$$


Заметим, что $\varphi^{(1)}(T)=y$. Ввиду (11) и марковского свойства мы получаем, что

$$
\begin{aligned}
& \liminf _{n \rightarrow \infty} n^{-1} \ln \mathbf{P}\left\{\delta_{n} \notin D_{\theta}\right\}=\liminf _{n \rightarrow \infty} n^{-1} \ln \mathbf{P}_{0,0}\left\{Y(s(n)) \notin D_{\theta}\right\} \\
& \geqslant \liminf _{n \rightarrow \infty} \inf _{y \in[-K, K]^{p}} n^{-1} \ln \mathbf{P}_{s(n)+T, y}\left\{\left\|Y-\varphi^{(1)}\right\|_{\infty}<\varepsilon_{0}\right\} \\
& \geqslant-I_{(T, 0)}\left(\varphi^{(1)}\right)=-\left(I_{(T, T / 2)}\left(\varphi^{(1)}\right)+I_{(T / 2,0)}\left(\varphi^{(0)}\right)\right) \\
& \geqslant-\left(\int_{T}^{T / 2} e^{t} H\left(\dot{\varphi}^{(1)}, \varphi^{(1)}\right) d t+\inf _{x \notin D_{\theta}} V_{\theta}(x)+\varepsilon\right) \\
& \geqslant-\left(\inf _{x \notin D_{\theta}} V_{\theta}(x)+\varepsilon+h_{0} e^{T / 2}\right)
\end{aligned}
$$

где $h_{0}=\max \left\{H_{\theta}(u, \vartheta): u \in[-4 K /|T|, 4 K /|T|]^{p}, \vartheta \in[-K, K]^{p}\right\}$. Выберем $|T|$ столь большим, чтобы $h_{0} e^{T / 2}<\varepsilon$. Поскольку $\varepsilon$ произвольно мало, отсюда следует нижняя граница в (5).

Для доказательства верхней границы в (5) введем следующие множества. Для фиксированного $T, T<0$, положительного $\lambda$ и $y \in[-K, K]^{p}$ положим

$$
W_{\theta}^{(T, y)}(\lambda)=\left\{x: \inf _{\varphi(T)=y, \varphi \in \Phi(x)} I_{(T, 0)} \leqslant \lambda\right\} .
$$

Как показано в [14] для сколь угодно малого $\varepsilon_{0}>0$ справедлива верхняя граница

$$
\limsup _{n \rightarrow \infty} n^{-1} \ln \mathbf{P}_{s(n)+T, y}\left\{\operatorname{dist}\left(Y(s(n)) ; W_{\theta}^{(T, y)}(\lambda)\right) \geqslant \varepsilon_{0}\right\} \leqslant-\lambda .
$$

Возьмем сколь угодно малое положительное значение $\varepsilon$. Введем

$$
\lambda_{0}=\inf _{x \notin D_{\theta}} V_{\theta}(x)>0, \quad \varepsilon_{0}=\operatorname{dist}\left(D_{\theta}^{c} ; W_{\theta}\left(\lambda_{0}-\varepsilon\right)\right)>0 .
$$

Далее, для любого $\varepsilon$ существует столь большое $|T|$, что

$$
W_{\theta}^{(T, y)}\left(\lambda_{0}-2 \varepsilon\right) \subseteq W_{\theta}\left(\lambda_{0}-\varepsilon\right)
$$

Действительно, если $x \in W_{\theta}^{(T, y)}\left(\lambda_{0}-2 \varepsilon\right)$, то тогда существует $\varphi_{*}^{(0)}: \varphi_{*}^{(0)}(T)=y \in[-K, K]^{p}, \varphi_{*}^{(0)}(0)=x$ и $I_{(T, 0)}\left(\varphi_{*}^{(0)}\right) \leqslant \lambda_{0}-2 \varepsilon+\varepsilon / 2$. Положим

$$
\varphi_{*}^{(1)}(t)= \begin{cases}\varphi_{*}^{(0)}(t), & \text { если } t \in[T, 0], \\ \theta+\left(\varphi_{*}^{(0)}(T)-\theta\right)(t-T+1), & \text { если } t \in[T-1, T] \\ \theta, & \text { если } t \in(-\infty, T-1] .\end{cases}
$$

Аналогично доказательству нижней границы, можно найти столь большое $|T|$, что

$$
\inf _{\varphi \in \Phi(x)} I_{(-\infty, 0)}(\varphi) \leqslant I_{(-\infty, 0)}\left(\varphi_{*}^{(1)}\right) \leqslant I_{(T, 0)}\left(\varphi_{*}^{(0)}\right)+\frac{\varepsilon}{2} \leqslant \lambda_{0}-\varepsilon .
$$


Поскольку $\varphi_{*}^{(1)}(0)=x$, постольку последнее неравенство влечет $x \in W_{\theta}\left(\lambda_{0}-\varepsilon\right)$, откуда следует (13). Итак,

$$
\begin{aligned}
\left\{Y(s(n)) \in D_{\theta}^{c}\right\} & \subseteq\left\{\operatorname{dist}\left(Y(s(n)) ; W_{\theta}\left(\lambda_{0}-\varepsilon\right)\right) \geqslant \varepsilon_{0}\right\} \\
& \subseteq\left\{\operatorname{dist}\left(Y(s(n)) ; W_{\theta}^{(T, y)}\left(\lambda_{0}-2 \varepsilon\right)\right) \geqslant \varepsilon_{0}\right\} .
\end{aligned}
$$

Применяя (12) и марковское свойство, мы получаем верхнюю границу:

$$
\begin{aligned}
& \limsup _{n \rightarrow \infty} n^{-1} \ln \mathbf{P}\left\{\delta_{n} \notin D_{\theta}\right\}=\limsup _{n \rightarrow \infty} n^{-1} \ln \mathbf{P}_{0,0}\left\{Y(s(n)) \in D_{\theta}^{c}\right\} \\
& \quad \leqslant \limsup _{n \rightarrow \infty} \sup _{y \in[-K, K]^{p}} n^{-1} \ln \mathbf{P}_{s(n)+T, y}\{\operatorname{dist}(Y(s(n)) ; \\
& \left.\left.\quad W_{\theta}^{(T, y)}\left(\lambda_{0}-2 \varepsilon\right)\right) \geqslant \varepsilon_{0}\right\} \\
& \leqslant-\left(\lambda_{0}-2 \varepsilon\right)=-\inf _{x \notin D_{\theta}} V_{\theta}(x)+2 \varepsilon .
\end{aligned}
$$

Теорема доказана.

Д оказательст во предложения 1. В пространстве вектор-функции $\varphi$ таких, что $\int_{-\infty}^{0} e^{t} \varphi^{T}(t) \varphi(t) d t<\infty$, мы введем скалярное произведение

$$
\left\langle\varphi_{1}, \varphi_{2}\right\rangle_{F}=\int_{-\infty}^{0} e^{t} \varphi_{1}^{T}(t) F^{-1} \varphi_{2}(t) d t
$$

и норму $\|\varphi(t)\|_{F}^{2}=\langle\varphi, \varphi\rangle_{F}$. Преобразование Лежандра правой части (6) имеет вид:

$$
H_{\theta}(u, \vartheta)=\frac{1}{2}[u+\mathscr{K}(\vartheta-\theta)]^{T} F^{-1}[u+\mathscr{K}(\vartheta-\theta)]
$$

Итак, в данном случае функционал действия $I_{(\tau, 0)}(\varphi)=\frac{1}{2} \| \dot{\varphi}(t)+$ $\mathscr{K}(\varphi(t)-\theta) \|_{F}^{2}$, и квазипотенциал превращается в

$$
V_{\theta}(x)=\inf \left\{\frac{1}{2}\|\dot{\varphi}(t)+\mathscr{K}(\varphi(t)-\theta)\|_{F}^{2}, \varphi(0)=x\right\} .
$$

Положим $\psi(t)=\dot{\varphi}(t)+\mathscr{K}(\varphi(t)-\theta)$. Тогда $\varphi(t)-\theta=e^{-t \mathscr{K}} \times$ $\int_{-\infty}^{t} e^{s \mathscr{K}} \psi(s) d s$, и

$$
V_{\theta}(x)=\inf \left\{\frac{1}{2}\|\psi\|_{F}^{2}, \int_{-\infty}^{0} e^{t \mathscr{K}} \psi(t) d t=x-\theta\right\} .
$$

Обозначим $i$-ю строку матрицы $\exp \{t(\mathscr{K}-\mathrm{I})\} F$ через $\mathrm{e}_{i}^{T}(t)$ и положим $y=x-\theta=\left(y_{1}, \ldots, y_{p}\right)^{T}$. В этих обозначениях

$$
V_{\theta}(x)=\inf \left\{\frac{1}{2}\|\psi\|_{F}^{2}, y_{i}=\left\langle\mathbf{e}_{i}^{T}(t), \psi\right\rangle_{F}, i=1, \ldots, p\right\}
$$


Задача минимизации (14) допускает решение в форме $\psi(t)=$ $\sum_{j=1}^{p} \alpha_{j} \mathbf{e}_{j}(t)$. Здесь $\alpha=\left(\alpha_{1}, \ldots, \alpha_{p}\right)^{T}$ удовлетворяет системе линейных уравнений $\mathscr{f} \alpha=y$. Окончательно мы получаем, что $\psi(t)=$ $F \exp \{t(\mathscr{K}-\mathbf{I})\} \mathscr{S}^{-1} y$ и

$$
\begin{aligned}
V_{\theta}(x) & =\frac{1}{2} y^{T} \mathscr{S}^{-1}\left[\int_{-\infty}^{0} \exp \{t(\mathscr{K}-\mathbf{I})\} F\left(e^{t} F^{-1}\right) F \exp \{t(\mathscr{K}-\mathbf{I})\} d t\right] \mathscr{S} y \\
& =\frac{1}{2} y^{T} \mathscr{S}^{-1} y=\frac{1}{2}(x-\theta)^{T} \mathscr{S}^{-1}(x-\theta) .
\end{aligned}
$$

Предложение доказано.

Для доказательства предложения 2 нам потребуется лемма.

Лемма. (i) Минимум по а квадратичной формы

$$
B(\alpha)=\left(z_{1}-\alpha, \bar{z}^{T}\right) \Sigma^{-1}\left(\begin{array}{c}
z_{1}-\alpha \\
\bar{z}
\end{array}\right)
$$

достигаетсл при $\alpha_{*}=z_{1}-\sigma_{1} \mathbf{s}^{T} \bar{z}$ и равен $\bar{z}^{T} \bar{F}^{-1} \bar{z}$.

(ii) Ecлu $z_{j}=\sigma_{j} \rho_{j} z_{1} / \sigma_{1}$ npu $j=2, \ldots, p$, mo $B=B(0)=z^{T} \Sigma^{-1} z=$ $z_{1}^{2} / \sigma_{1}^{2}$.

(iii) $B=z^{T} \Sigma^{-1} z \geqslant z_{1}^{2} / \sigma_{1}^{2}$.

(iv) $B=z^{T} \Sigma^{-1} z \geqslant \sum_{2}^{p}\left(z_{j}^{2} / \sigma_{j}^{2}\right) \bar{z}^{T} \bar{F}^{-1} \bar{z}$.

Доказ ат ельство лем мы. (i) Заметим, что обратная матрица $\Sigma^{-1}$ может быть записана как

$$
\Sigma^{-1}=\left(\begin{array}{cccc}
\frac{1}{\sigma_{1}^{2}\left(1-R^{2}\right)} & -\frac{\rho_{2}}{\sigma_{1} \sigma_{2}\left(1-R^{2}\right)} & \cdots & -\frac{\rho_{p}}{\sigma_{1} \sigma_{p}\left(1-R^{2}\right)} \\
-\frac{\rho_{2}}{\sigma_{1} \sigma_{2}\left(1-R^{2}\right)} & \frac{1}{\sigma_{2}^{2}}+\frac{\rho_{2}^{2}}{\sigma_{2}^{2}\left(1-R^{2}\right)} & \cdots & \frac{\rho_{2} \rho_{p}}{\sigma_{2} \sigma_{p}\left(1-R^{2}\right)} \\
\cdots & \cdots & \cdots & \cdots \\
-\frac{\rho_{p}}{\sigma_{1} \sigma_{p}\left(1-R^{2}\right)} & \frac{\rho_{2} \rho_{p}}{\sigma_{2} \sigma_{p}\left(1-R^{2}\right)} & \cdots & \frac{1}{\sigma_{p}^{2}}+\frac{\rho_{p}^{2}}{\sigma_{p}^{2}\left(1-R^{2}\right)}
\end{array}\right) .
$$

Итак,

$$
\begin{aligned}
B(\alpha)= & \frac{\left(z_{1}-\alpha\right)^{2}}{\sigma_{1}^{2}\left(1-R^{2}\right)}-2\left(z_{1}-\alpha\right) \sum_{2}^{p} \frac{\rho_{j} z_{j}}{\sigma_{1} \sigma_{j}\left(1-R^{2}\right)} \\
& +\frac{1}{1-R^{2}}\left(\sum_{2}^{p} \frac{\rho_{j} z_{j}}{\sigma_{j}}\right)^{2}+\sum_{2}^{p}\left(\frac{z_{j}}{\sigma_{j}}\right)^{2}
\end{aligned}
$$

Дифференцируя последнюю формулу по $\alpha$, мы находим точку минимума

$$
\alpha_{*}=z_{1}-\sigma_{1} \sum_{2}^{p} \frac{\rho_{j} z_{j}}{\sigma_{j}}=z_{1}+\sigma_{1}^{2}\left(1-R^{2}\right) \mathbf{s}^{T} \bar{z} .
$$


Непосредственные вычисления показывают, что $B\left(\alpha_{*}\right)=\sum_{2}^{p}\left(z_{j} / \sigma_{j}\right)^{2}=$ $\bar{z}^{T} \bar{F}^{-1} \bar{z}$.

(ii) Положим $z_{j}=\sigma_{j} \rho_{j} z_{1} / \sigma_{1}, \alpha=0$. Тогда

$$
\begin{aligned}
B=B(0) & =\frac{z_{1}^{2}}{\sigma_{1}^{2}\left(1-R^{2}\right)}\left(1-2 \sum_{2}^{p} \rho_{j}^{2}+\left(\sum_{2}^{p} \rho_{j}^{2}\right)^{2}\right)+\frac{z_{1}^{2}}{\sigma_{1}^{2}} \sum_{2}^{p} \rho_{j}^{2} \\
& =\frac{z_{1}^{2}}{\sigma_{1}^{2}}\left(\frac{1-2 R^{2}+R^{4}}{1-R^{2}}+R^{2}\right)=\frac{z_{1}^{2}}{\sigma_{1}^{2}} .
\end{aligned}
$$

(iii) Имеем

$$
B=\frac{z_{1}^{2}}{\sigma_{1}^{2}}+\left(1-R^{2}\right)^{-1}\left[\sum_{2}^{p}\left(\frac{z_{1} \rho_{j}}{\sigma_{1}}-\frac{z_{j}}{\sigma_{j}}\right)^{2}+\left(\sum_{2}^{p} \frac{z_{j} \rho_{j}}{\sigma_{j}}\right)^{2}-R^{2} \sum_{2}^{p} \frac{z_{j}^{2}}{\sigma_{j}^{2}}\right] .
$$

Минимум последней квадратичной формы достигается при $z_{1} / \sigma_{1}=$ $R^{-2} \sum_{2}^{p} z_{j} \rho_{j} / \sigma_{j}$ и равен

$$
R^{-2}\left(1-R^{2}\right)\left[R^{2} \sum_{2}^{p} \frac{z_{j}^{2}}{\sigma_{j}^{2}}-\left(\sum_{2}^{p} \frac{z_{j} \rho_{j}}{\sigma_{j}}\right)^{2}\right] \geqslant 0 .
$$

(iv) Из (iii) следует, что

$$
B=\sum_{2}^{p} \frac{z_{j}^{2}}{\sigma_{j}^{2}}+\left(1-R^{2}\right)^{-1}\left(\frac{z_{1}}{\sigma_{1}}-\sum_{2}^{p} \frac{z_{j} \rho_{j}}{\sigma_{j}}\right)^{2} .
$$

Лемма доказана.

Доказательство предложения 2. По определению имеем

$$
H_{\theta}(u, \vartheta)=\frac{1}{2}\left(u_{1}+a_{1}\left(\vartheta_{1}+1\right),(\bar{u}+A(\bar{v}-\bar{\theta}))^{T}\right) \Sigma^{-1}\left(\begin{array}{c}
u_{1}+a_{1}\left(\vartheta_{1}+1\right) \\
\bar{u}+A(\bar{v}-\bar{\theta})
\end{array}\right)
$$

и $\widetilde{H}_{\theta}(u, \vartheta)=H_{\theta}(u, \vartheta)$, если или $u_{1}>0$, или $\vartheta_{1}>0$. Далее, если $u_{1}=$ $\vartheta_{1}=0$ и $a_{1}-\sigma_{1} \mathbf{s}^{T}(\bar{u}+A(\bar{v}-\bar{\theta}))<0$, тогда все еще $\widetilde{H}_{\theta}(u, \vartheta)=H_{\theta}(u, \vartheta)$. Однако, если $a_{1}-\sigma_{1} \mathbf{s}^{T}(\bar{u}+A(\bar{v}-\bar{\theta})) \geqslant 0$, то

$$
H_{\theta}(u, \vartheta)=\widetilde{H}_{\theta}((0, \bar{u}),(0, \bar{\vartheta}))=\frac{1}{2}(\bar{u}+A(\bar{v}-\bar{\theta}))^{T} \bar{F}^{-1}(\bar{u}+A(\bar{v}-\bar{\theta})) .
$$

Доказательство (15) немедленно вытекает из п. (i) леммы при $z_{1}=a_{1}$. Важно отметить, что при $\varphi_{1}(t)=0, \bar{\varphi}(t) \approx \bar{\theta}$ и малых $\bar{u}=\dot{\bar{\varphi}}(t)$ функционал действия $\tilde{I}_{(-\infty, 0)}(\varphi)$ совпадает с функционалом действия, определяемым маргинальным распределением последних $p-1$ координат независимо от значения s. Более точно, для таких функций $\varphi$ при $\lambda \rightarrow 0$ выполнено

$$
\tilde{I}_{(-\infty, 0)}(\varphi) \sim \frac{1}{2} \int_{-\infty}^{0} e^{t}[\dot{\bar{\varphi}}(t)+A(\bar{\varphi}(t)-\bar{\theta})]^{T} \bar{F}^{-1}[\dot{\bar{\varphi}}(t)+A(\bar{\varphi}(t)-\bar{\theta})] d t
$$


и, следовательно, применимо предложение 1. Эти соображения объясняют происхождение второго слагаемого в левой части (10).

Верхняя граница. Необходимо убедиться, что для любого $x$, удовлетворяющего (10), имеет место неравенство

$$
\tilde{V}_{(0, \bar{\theta})}(x) \leqslant \lambda\left[1+o_{\lambda}(1)\right] \text { при } \lambda \rightarrow 0 .
$$

Заметим, что если $x_{1}$ и $\bar{x}$ удовлетворяют $(10)$, то $x_{1}=O(\lambda)$ и $\|\bar{x}-\bar{\theta}\|=$ $O(\sqrt{\lambda})$, где $\|\cdot\|-$ евклидова норма. Положим $t_{0}=-x_{1} / a_{1}$, так что $\left|t_{0}\right|=O(\lambda)$ при $\lambda \rightarrow 0$.

Найдем траекторию $\varphi^{*}=\varphi^{*}(t),\left(\varphi^{*}\right)^{T}=\left(\varphi_{1}^{*},\left(\bar{\varphi}^{*}\right)^{T}\right)$, которая обеспечивает верхнюю границу квазипотенциала. А именно выберем

$$
\varphi_{1}^{*}(t)=0, \quad \text { если } t \leqslant t_{0}, \quad \text { и } \quad \varphi_{1}^{*}(t)=a_{1} t+x_{1} \quad \text { в противном случае. }
$$

Пусть компоненты $\varphi_{j}^{*}(t)$ выбраны так, что для всех $j=2, \ldots, p$ выполнено

$$
\begin{aligned}
& {\left[\dot{\bar{\varphi}}^{*}(t)+\left[A\left(\bar{\varphi}^{*}(t)-\bar{\theta}\right)\right]\right]_{j}} \\
& \quad=\frac{\sigma_{j} \rho_{j}}{\sigma_{1}}\left[\dot{\varphi}_{1}^{*}(t)+a_{1}\left(\varphi_{1}^{*}(t)+1\right)\right], \quad \text { когда } t_{0}<t \leqslant 0,
\end{aligned}
$$

с конечным условием $\bar{\varphi}^{*}(0)=\left(\varphi_{2}^{*}(0), \ldots, \varphi_{p}^{*}(0)\right)^{T}=\bar{x}$. Положим $\bar{x}_{0}=\bar{\varphi}^{*}\left(t_{0}\right)$. Как легко видеть из $(17),\left\|\bar{x}_{0}-\bar{x}\right\|=O\left(\left|t_{0}\right|\right)=O(\lambda)$. Следовательно,

$$
\left\|\left(\bar{x}_{0}-\bar{\theta}\right)-(\bar{x}-\bar{\theta})\right\|=O(\lambda) \quad \text { и } \quad\left\|\bar{x}_{0}-\bar{\theta}\right\|=O(\sqrt{\lambda}) \quad \text { при } \lambda \rightarrow 0
$$

Определим $\bar{\varphi}^{*}(t)$ для $t \leqslant t_{0}$ с помощью дифференциального уравнения

$$
\bar{\psi}(t)=\left[\dot{\bar{\varphi}}^{*}(t)+A\left(\bar{\varphi}^{*}(t)-\bar{\theta}\right)\right]=\bar{F} e^{\left(t-t_{0}\right)(A-I) \overline{\mathscr{S}}^{-1}}\left(\bar{x}_{0}-\bar{\theta}\right)
$$

с начальным условием $\bar{\varphi}^{*}(-\infty)=0$. Для достаточно малых $\lambda$ (ср. замечание 4) мы имеем

$$
\begin{aligned}
a_{1}-\sigma_{1} \mathbf{s}^{T}\left[\dot{\bar{\varphi}}^{*}(t)+A\left(\bar{\varphi}^{*}(t)-\bar{\theta}\right)\right] & =a_{1}-\sigma_{1} \mathbf{s}^{T} \bar{\psi}(t)=a_{1}+O\left(\left\|\bar{x}_{0}-\bar{\theta}\right\|\right) \\
& =a_{1}+O(\sqrt{\lambda}) \geqslant 0
\end{aligned}
$$

Далее, вычислим значение функционала на траектории $\varphi^{*}(t)$ :

$$
\tilde{V}_{\theta}(x) \leqslant \tilde{I}_{(-\infty, 0)}\left(\varphi^{*}\right)=\int_{-\infty}^{t_{0}} e^{t} \tilde{H}_{\theta}\left(\dot{\varphi}^{*}(t), \varphi^{*}(t)\right) d t+\int_{t_{0}}^{0} e^{t} \tilde{H}_{\theta}\left(\dot{\varphi}^{*}(t), \varphi^{*}(t)\right) d t
$$


Применяя п. (ii) леммы, мы получаем

$$
\begin{aligned}
\int_{t_{0}}^{0} e^{t} \widetilde{H}_{\theta}\left(\dot{\varphi}^{*}(t), \varphi^{*}(t)\right) d t & =\frac{1}{2 \sigma_{1}^{2}} \int_{t_{0}}^{0} e^{t}\left(\dot{\varphi}_{1}{ }^{*}(t)+a_{1}\left(\varphi^{*}(t)+1\right)\right)^{2} d t \\
& =\frac{1}{2 \sigma_{1}^{2}} \int_{t_{0}}^{0} e^{t}\left(2 a_{1}+a_{1}^{2} t+a_{1} x_{1}\right)^{2} d t \\
& =\frac{4 a_{1}^{2}\left|t_{0}\right|(1+O(\lambda))}{2 \sigma_{1}^{2}}=\frac{2 a_{1} x_{1}(1+O(\lambda))}{\sigma_{1}^{2}}
\end{aligned}
$$

где было использовано тождество $a_{1}\left|t_{0}\right|=x_{1}$. Ввиду (15), (18) и (19), как и в предложении 1 ,

$$
\begin{aligned}
\int_{-\infty}^{t_{0}} e^{t} \widetilde{H}_{\theta}\left(\dot{\varphi}^{*}(t), \varphi^{*}(t)\right) d t & =\frac{1}{2} \int_{-\infty}^{t_{0}} e^{t} \bar{\psi}^{T}(t) F^{-1} \bar{\psi}(t) d t \\
& =\frac{e^{t_{0}}}{2}\left(\bar{x}_{0}-\bar{\theta}\right)^{T} \overline{\mathscr{S}}^{-1}\left(\bar{x}_{0}-\bar{\theta}\right) \\
& =\frac{1}{2}(\bar{x}-\bar{\theta})^{T} \overline{\mathscr{S}}^{-1}(\bar{x}-\bar{\theta})[1+O(\sqrt{\lambda})] .
\end{aligned}
$$

Комбинируя (20) с (21), получаем неравенство $\tilde{V}_{\theta}(x) \leqslant \lambda+O\left(\lambda^{3 / 2}\right)$ при $\lambda \rightarrow 0$, которое и доказывает верхнюю границу.

Нижняя гранииа. Для вывода нижней границы рассмотрим траекторию $\varphi(t), t \leqslant 0, \varphi^{T}=\left(\varphi_{1}, \bar{\varphi}^{T}\right)$, которая заканчивается в $x$, т.е. $\varphi_{1}(0)=x_{1}, \bar{\varphi}(0)=\bar{x}$. При условии, что $x$ удовлетворяет (10), мы хотим показать, что $\tilde{I}_{(-\infty, 0)}(\varphi)$ превосходит $\lambda\left(1+o_{\lambda}(1)\right)$. Введем $t_{*}=$ $\sup \left\{t: \varphi_{1}(t)=0\right\}$ с $t_{*}=-\infty$, если $\varphi_{1}(t)>0$ для всех $t$. В соответствии с п. (iii) леммы,

$$
\begin{aligned}
\tilde{I}_{(-\infty, 0)}(\varphi) & \geqslant \frac{1}{2 \sigma_{1}^{2}} \int_{t_{*}}^{0} e^{t}\left[\dot{\varphi}_{1}(t)+a_{1}\left(\varphi_{1}(t)+1\right)\right]^{2} d t \\
& \geqslant \operatorname{linf}_{\varphi_{1}\left(t_{*}\right)=0, \varphi_{1}(0)=x_{1}, \varphi_{1}(t)>0} \int_{t_{*}}^{0} \frac{e^{t}}{2 \sigma_{1}^{2}}\left[\dot{\varphi}_{1}(t)+a_{1}\left(\varphi_{1}(t)+1\right)\right]^{2} d t \\
& =J_{*} .
\end{aligned}
$$

Для того чтобы оценить $J_{*}$ в (22) снизу, решим уравнение Эйлера. Из уравнения Эйлера следует, что любое решение экстремальной задачи выше удовлетворяет дифференциальному уравнению $\ddot{\varphi}_{1}(t)+\dot{\varphi}_{1}(t)+$ $a_{1}\left(1-a_{1}\right)\left[\varphi_{1}(t)+1\right]=0 \mathrm{c}$ общим решением $\varphi_{1}(t)=p_{1} e^{\left(a_{1}-1\right) t}+p_{2} e^{-a_{1} t}-1$ и граничными условиями $\varphi_{1}\left(t_{*}\right)=0, \varphi_{1}(0)=x_{1}$. Отсюда получается, что экстремаль в $(22)$ имеет вид $\dot{\varphi}_{1}(t)+a_{1}\left[\varphi_{1}(t)+1\right]=p_{1}\left(2 a_{1}-1\right) e^{\left(a_{1}-1\right) t}$ c $p_{1}=\left(1+x_{1}-e^{a_{1} t_{*}}\right) /\left(1-e^{\left(2 a_{1}-1\right) t_{*}}\right)$. Итак, в (22)

$$
J_{*}=\int_{t_{*}}^{0} \frac{e^{t}}{2 \sigma_{1}^{2}}\left[\dot{\varphi}_{1}(t)+a_{1}\left(\varphi_{1}(t)+1\right)\right]^{2} d t=\frac{\left(2 a_{1}-1\right)\left[1+x_{1}-e^{a_{1} t_{*}}\right]^{2}}{2 \sigma_{1}^{2}\left(1-e^{\left(2 a_{1}-1\right) t_{*}}\right)}
$$


Для минимизации правой части (23) следует выбрать $\left|t_{*}\right|=O(\lambda)$, что влечет

$$
J_{*}=\frac{1}{2 \sigma_{1}^{2}}\left(\frac{x_{1}^{2}}{\left|t_{*}\right|}+2 a_{1} x_{1}+a_{1}^{2}\left|t_{*}\right|+O\left(\lambda^{2}\right)\right) \geqslant \frac{2 a_{1} x_{1}}{\sigma_{1}^{2}}+O\left(\lambda^{2}\right) .
$$

Для любого $\delta, 0<\delta<1$, положим $\omega_{\delta}(t)=1$ при $t \leqslant t_{*}$ и $\omega_{\delta}(t)=\delta$ при $t_{*}<t \leqslant 0$ и заметим, что

$$
\tilde{I}_{(-\infty, 0)}(\varphi)=(1-\delta) \int_{t_{*}}^{0} e^{t} \widetilde{H}(\dot{\varphi}, \varphi) d t+\int_{-\infty}^{0} \omega_{\delta}(t) e^{t} \widetilde{H}(\dot{\varphi}, \varphi) d t
$$

Применяя п. (iii) леммы к первому из этих интегралов и п. (iv) леммы ко второму, мы получаем неравенство

$$
\begin{aligned}
\tilde{I}_{(-\infty, 0)}(\varphi) \geqslant & \frac{1-\delta}{2 \sigma_{1}^{2}} \int_{t_{*}}^{0} e^{t}\left[\dot{\varphi}_{1}(t)+a_{1}\left(\varphi_{1}(t)+1\right)\right]^{2} d t \\
& +\frac{1}{2} \int_{-\infty}^{0} \omega_{\delta}(t) e^{t}[\dot{\bar{\varphi}}(t)+A(\bar{\varphi}(t)-\bar{\theta})]^{T} \bar{F}^{-1} \\
& \times[\dot{\bar{\varphi}}(t)+A(\bar{\varphi}(t)-\bar{\theta})] d t
\end{aligned}
$$

Как и в предложении 1 , второй интеграл представляет норму при скалярном произведении

$$
\langle\bar{\varphi}, \bar{\psi}\rangle=\int_{-\infty}^{0} w_{\delta} e^{t} \bar{\varphi}^{T} \bar{F}^{-1} \bar{\psi} d t
$$

и его минимум по $\bar{\varphi}$ таким, что $\bar{\varphi}(-\infty)=0$ и $\bar{\varphi}(0)=\bar{x}$, достигается, если

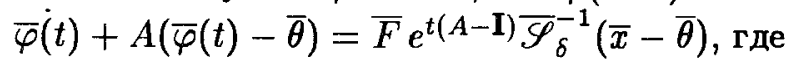

$$
\overline{\mathscr{S}}_{\delta}=\int_{-\infty}^{0} \omega_{\delta}^{-1}(t) e^{t} \exp \{t(A-\mathbf{I})\} \bar{F} \exp \{t(A-\mathbf{I})\} d t=\overline{\mathscr{S}}\left(1+O\left(\frac{\lambda}{\delta}\right)\right) .
$$

Значение этого минимума равно $\frac{1}{2}(\bar{x}-\bar{\theta})^{T} \overline{\mathscr{S}}^{-1}(\bar{x}-\bar{\theta})(1+O(\lambda / \delta))$. Выберем $\delta=\sqrt{\lambda}$. Если $x$ удовлетворяет $(10)$, тогда из (23)-(25) получается следующее неравенство:

$$
\begin{aligned}
\tilde{I}_{(-\infty, 0)}(\varphi) \geqslant & (1-\sqrt{\lambda})\left(\frac{2 a_{1} x_{1}}{\sigma_{1}^{2}}+O\left(\lambda^{2}\right)\right) \\
& +\frac{1}{2}(\bar{x}-\bar{\theta})^{T \bar{S}^{-1}}(\bar{x}-\bar{\theta})(1+O(\sqrt{\lambda}))=\lambda+O\left(\lambda^{3 / 2}\right) .
\end{aligned}
$$

Это завершает доказательство. 


\section{СПИСОК ЛИТЕРАТУРЫ}

1. Bahadur R. R. Some Limit Theorems in Statistics. Philadelphia, PA: SIAM, 1971, $42 \mathrm{p}$.

2. Dupuis $P$., Ellis R. S. A Weak Convergence Approach to the Theory of Large Deviations. New York: Wiley, 1997, 479 p.

3. Fabian $V$. On asymptotic normality in stochastic approximation. - Ann. Math. Statist., 1968, v. 39, p. 1327-1332.

4. Фрейдлин $М$. И. Функционал действия для одного класса случайных процессов. - Теория вероятн. и ее примен., 1972, т. 17, в. 3, с. 536-541.

5. Вентиель $A$. Д., Фрейдлия $M$. И. Флуктуации в динамических системах под действием малых случайных возмущений. М.: Наука, 1979.

6. Коростелев А. П. Стохастические рехуррентные процедуры. М.: Наука, 1984.

7. Коростелев $A$. П., Леонов $C$. Л. Функционал действия для диффузионного процесса с разрывным переносом. - Теория вероятн. и ее примен., 1992, v. 37, в. 3, c. $543-550$.

8. Korostelev A. P., Leonov S. L. Action functional for diffusions in discontinuous media. - Probab. Theory Relat. Fields, 1993, v. 94, p. 317-333.

9. Korostelev A. P., Rukhin A. L. Large deviations probabilities for recursive M-estimators. - Stochastics Stochastics Rep., 1996, v. 57, p. 185-197.

10. Невельсон М. Б., Хасьминский $P$. 3. Стохастическая аппроксимация и рекуррентное оценивание. М.: Наука, 1972, 308 c.

11. Rukhin A. L. Recursive testing of multiple hypotheses: Consistency and efficiency of the Bayes rule. - Ann. Statist., 1994, v. 22, p. 616-633.

12. Sacks J. Asymptotic distributions of stochastic approximation. - Ann. Math. Statist., 1958, v. 29, p. 373-405.

13. Sakrison $D$. J. Efficient recursive estimation: Application to estimating the parameters of a covariance function. - Internat. J. Engrg. Sci., 1965, v. 3, p. 461-483.

14. Веятиель $A$. Д. Предельные теоремы о больших уклонениях для марковских случайных процессов. М.: Наука, 1986. 